\title{
Numerical Study of the Air Injection Method into the Flat-Panel Photobioreactor
}

\author{
Miroslav Rebej, Jiří Vondál, Zdeněk Jegla \\ Institute of Process Engineering, Faculty of Mechanical Engineering, Brno University of Technology \\ Technická 2896/2, 61669 Brno, Czech Republic \\ rebej@fme.vutbr.cz; vondal@fme.vutbr.cz; jegla@fme.vutbr.cz
}

\begin{abstract}
Computational Fluid Dynamics (CFD) is used to investigate the effect of a boundary condition on bubbly flow. The fluid domain is based on a section of a lab-scale photobioreactor where the gaseous phase is injected into the water. The paper presents two means of gaseous phase injection. The first method uses the mass-source term defined in a dedicated cell zone. The second approach follows the real-world setup where the air flows through the orifice modelled as a surface area on the aerator tube. Numerical results are analysed in terms of the equivalent diameter, bubble aspect ratio and specific dimensionless numbers. It was found that the size and shape of bubbles are independent of the boundary condition definition. However, the difference in the bubble rising velocity was observed. Furthermore, the position of the separation point and the size of the gaseous plume was found to be different among the studied cases.
\end{abstract}

Keywords: Multiphase flow, VOF model, Bubbly flow, Boundary condition

\section{Introduction}

Microalgae are naturally ecologically diverse and have the ability to adapt to different life conditions. Therefore, they can be found growing in many biotopes. They are also biological $\mathrm{CO}_{2} / \mathrm{O}_{2}$ exchangers and can offer a large biotechnological potential. However, the utilization of such biotechnology must be based on a strong biotechnological basis and the intended biomass utilization must also be taken into account when considering a technical solution. Photobioreactors, i.e., closed technical systems for the cultivation processes of microorganisms, give a clear advantage over open systems, such as ponds, when it comes to an intensive production of high-value bioproducts. Closed photobioreactors allow for cultivation under controlled conditions so that the medium does not get contaminated or lost due to evaporation. However, the cultivation process in such photobioreactors has different operational requirements as the living conditions of cells in closed vessels are often far from their natural habitat, e.g., higher cell densities in the medium resulting in issues with irradiation density and patterns, different variations of $\mathrm{pH}$ and temperature, and different cells' experience with shear stresses that can exceed potential death-levels due to induced flow velocities [1]. Therefore, the design of such technical solutions needs to be evaluated for a cost-effective application and economic feasibility. When an optimum growth is reached, all aspects of microalgae cultivation are in balance, i.e., hydrodynamics, mass transfer, irradiation, and cell growth [2].

The paper focuses on the multiphase hydrodynamics of a photobioreactor. The way air is injected into the domain through the injection orifices can have an impact on bubble sizes and mixing characteristics of the mixture. This can also affect the living conditions of cells as the nutrient availability can be reduced or it can create high shear-stress zones that are deadly for microalgae cells. It is expected that the most cell deaths occur at the inlet regions where bubbles are formed and separated from the nozzle [3]. The design of the sparger can be crucial for the performance of a photobioreactor. The objective of the paper is to study the effect of an air injection method on working parameters of a photobioreactor, e.g. bubble size and shape. The outcomes of the study are expected to help to design a numerical model of a complete photobioreactor.

\section{Problem Specification}

The content of a photobioreactor is a mixture of phases. The vessel is primarily filled with a liquid that serves as an environment for living cells, usually modelled as solids. In addition to that, a gaseous phase, air or $\mathrm{CO}_{2}$, flows through this mixture. Nevertheless, because the size of the microalgae is insignificant to the flow patterns, numerical analyses are often reduced to only two phases, the liquid and gaseous phases. The studied photobioreactor is a flat-panel stirred vessel described in more detail in [4]. However, for this paper, the domain is reduced to a section shown in Fig. 1. 


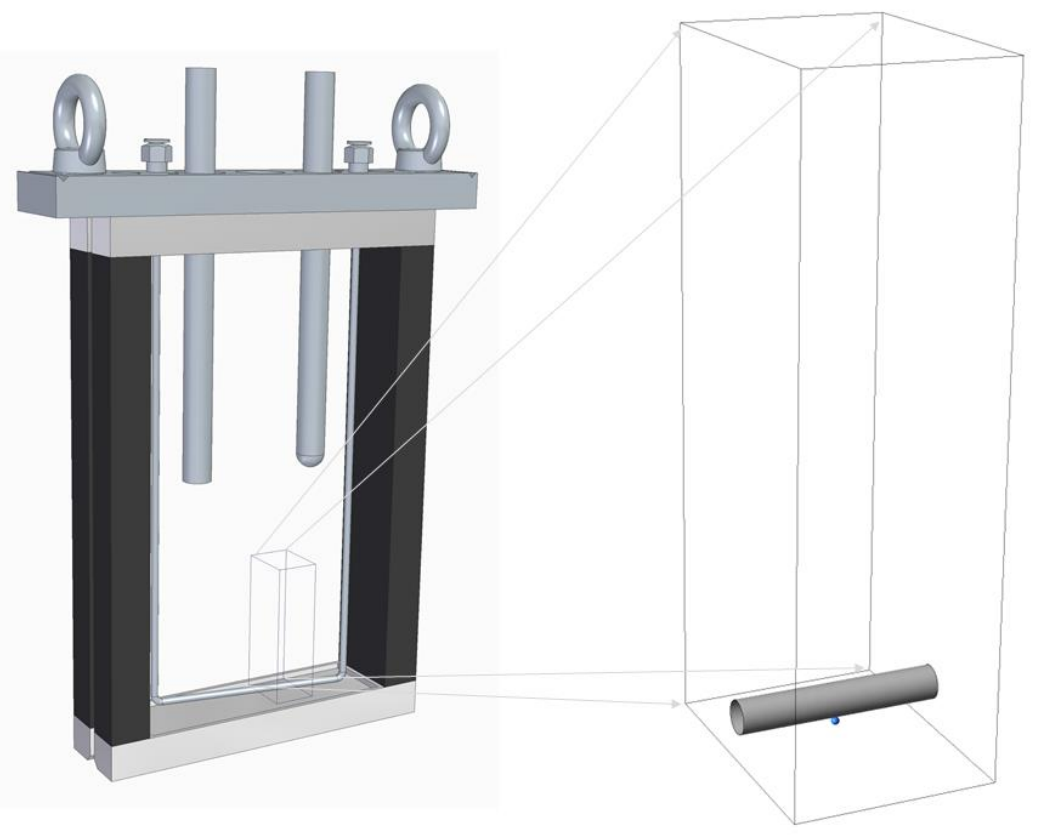

Fig. 1 Section of the domain

In the photobioreactor technology, the gaseous phase serves primarily as a nutrient for cells. However, it can also affect mixing of the medium [5]. Therefore, bubbles can have an impact on a variety of chemical and biochemical reactions that take place in the vessel [6]. Often, the strategy is to study bubbly flow at the level of a single bubble and apply the findings and set-up to subsequent, more complex, flow studies [7].

The bubbly flow is defined by means of the following dimensionless numbers: the Eötvös number (Eo), the Reynolds number $(R e)$, and the Morton number (Mo), Eqs. (1) - (3), [7].

$$
\begin{gathered}
E o=\frac{\Delta \rho g d_{B}^{2}}{\sigma} \\
R e=\frac{\rho_{c} v_{r e l} d_{B}}{\mu_{c}} \\
M o=\frac{g \mu_{c}^{4} \Delta \rho}{\rho_{c}^{2} \sigma^{3}}
\end{gathered}
$$

where $\Delta \rho$ is the density difference of the continuous and the dispersed phase, $g$ is the gravitational acceleration, $d_{B}$ is the bubble diameter, $\sigma$ is the surface tension, $\rho_{c}$ is the density of the continuous phase, $v_{r e l}$ is the relative velocity between phases, and $\mu_{c}$ is the dynamic viscosity of the continuous phase.

Depending on the medium and the flow regime, bubbles can have various forms of shapes and a problem can arise when defining the bubble size in terms of a single diameter. Hence, there are different approaches to determine the equivalent bubble diameter in the literature [7]-[10]. The equivalent bubble diameter $d_{B}$ considered in this work is defined in Eq. (4) by means of the horizontal and vertical diameter, $d_{h}$ and $d_{v}$ respectively. 


$$
d_{B}=\left(d_{h}^{2} d_{v}\right)^{1 / 3}
$$

\section{$3 \quad$ Numerical Model}

Under the normal operating conditions in the lab-scale photobioreactor, the air flow rate is set to $200 \mathrm{ml} / \mathrm{min}$ through four orifices with the diameter of $0.7 \mathrm{~mm}$. Using the section of the domain with only one inlet, the respective mass-flow-rate of air is $1.02 \mathrm{e}-6 \mathrm{~kg} / \mathrm{s}$.

Numerical analyses were assessed in the Eulerian-Eulerian framework in the ANSYS Fluent 2020R2 [11]. The domain was of a rectangular shape with the dimensions of $18 \times 24 \times 60 \mathrm{~mm}$. In the lower part, there was an aerator tube $(3 \mathrm{~mm}$ in diameter) going through the domain. The mesh consisted of 179,324 polyhedral cells with maximum cell length of $1 \mathrm{~mm}$. The numerical model simulated the injection of the secondary phase, air, into the primary phase, water.

In the first approach, air injection was modelled as a mass-source term in the spherical cell zone with the diameter of $0.7 \mathrm{~mm}$ and no velocity component. The second approach followed the real-world setup where the air was injected through the $0.7 \mathrm{~mm}$ orifice in the aerator tube. The applied mass-flow-rate boundary condition provided the air with the velocity of $1.75 \mathrm{~m} / \mathrm{s}$. The no-slip boundary condition was applied to walls corresponding to the walls of the photobioreactor. On the other hand, walls cutting the fluid domain of a photobioreactor were using the symmetry boundary condition. Next, at the top of the section, there was the pressure outlet boundary condition with no gauge pressure. The operating pressure of $101,325 \mathrm{~Pa}$ was specified at the level corresponding to the water level of the filled photobioreactor, i.e., $166 \mathrm{~mm}$ from the bottom surface. Respective geometries and boundary conditions are in Fig. 2.

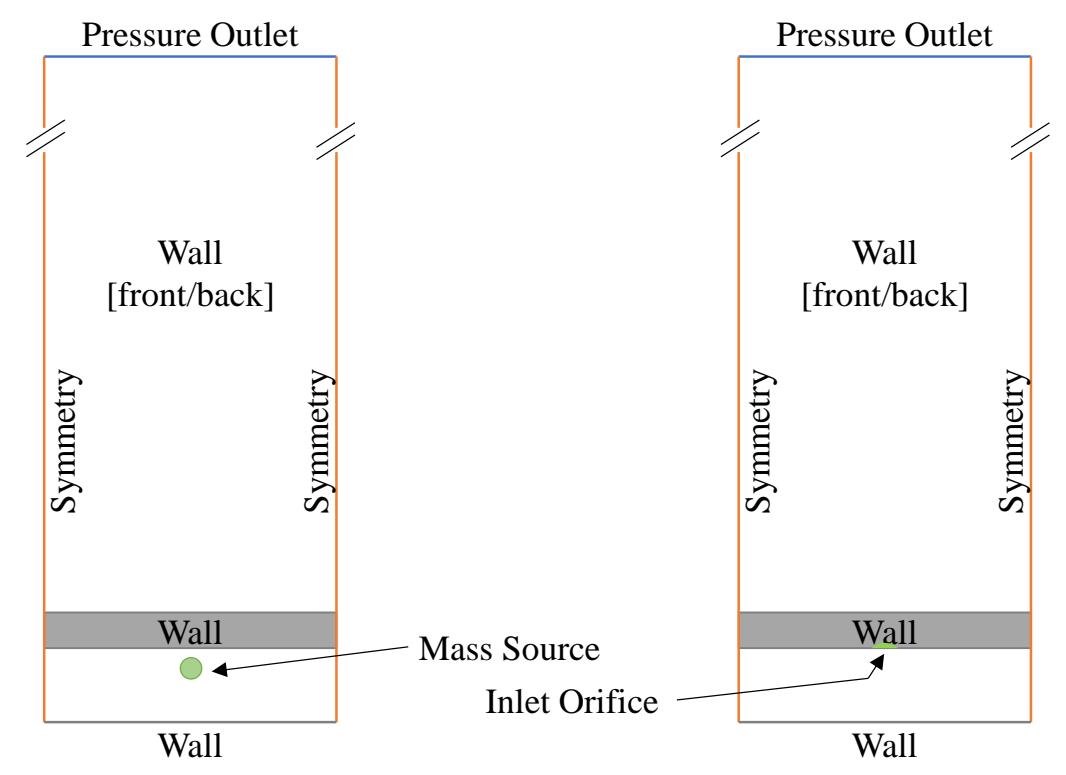

Fig. 2 Geometry and boundary conditions

\subsection{Volume of Fluid Model}

The multiphase flow in the PBR was simulated in the Volume of Fluid model (VOF). The VOF model allows numerical flow simulations of two or more immiscible fluids. The model tracks the phase interface using a single set of momentum equations and an additional volume fraction equation for each secondary phase. The continuity and momentum equations given in Eqs. (5) - (7) govern the flow of phases in the VOF model [11]: 


$$
\begin{gathered}
\frac{1}{\rho_{q}}\left[\frac{\partial}{\partial t}\left(\alpha_{q} \rho_{q}\right)+\nabla \cdot\left(\alpha_{q} \rho_{q} \vec{v}_{q}\right)=S_{\alpha_{q}}+\sum_{p=1}^{n}\left(\dot{m}_{p q}-\dot{m}_{q p}\right)\right] \\
\sum_{q=1}^{n} \alpha_{q}=1 \\
\frac{\partial}{\partial t}(\rho \vec{v})+\nabla \cdot(\rho \vec{v} \vec{v})=-\nabla p+\nabla \cdot\left[\mu\left(\nabla \vec{v}+\nabla \vec{v}^{T}\right)\right]+\rho \vec{g}+\vec{F}
\end{gathered}
$$

The numerical model includes the surface tension effects as they are expected to be important based on the Reynolds, $R e$, capillary, $C a$, and Weber number, $W e$ [11]. Their respective values are 1100 for $R e, 0.003$ for $C a$, and 3.4 for $W e$. Furthermore, the surface tension is modelled by the Constant Surface Force including the wall adhesion. The contact angle is set to $140^{\circ}$; surface tension coefficient is $0.072 \mathrm{~N} / \mathrm{m}$. The turbulence is modelled based on the Realizable $k-\varepsilon$ turbulence model with the enhanced wall treatment.

The simulations were carried out in the transient manner. The timestep size was set to $1 \mathrm{e}-5 \mathrm{~s}$ resulting in the Courant number of 2. The transient formulation was First Order Implicit. The spatial discretization is listed in Tab. 1. The Coupled scheme was used for the pressure-velocity coupling. The convergence criteria of 1e-3 were considered for all residuals in the simulation. Total flow-time that was simulated was $1.0 \mathrm{~s}$.

Tab. 1 Spatial Discretization

\begin{tabular}{lc}
\hline Gradient & Least Squares Cell Based \\
\hline Pressure & Body Force Weighted \\
\hline Momentum & Second Order Upwind \\
\hline Volume Fraction & Geo-Reconstruct \\
\hline Turbulent Kinetic Energy & Second Order Upwind \\
\hline Turbulent Dissipation Rate & Second Order Upwind \\
\hline
\end{tabular}

\section{Results}

The evaluation of the equivalent diameter and aspect ratio was based on the last three bubbles from the simulation time span. Bubbles passing the height level of $35 \mathrm{~mm}$ were evaluated. The height level was selected for the diameter evaluation as bubbles were already formed, shaped and not yet affected by the outlet boundary condition. Also, there was no coalescence/break-up observed at this height level (see Fig. 3 and Fig. 4). 


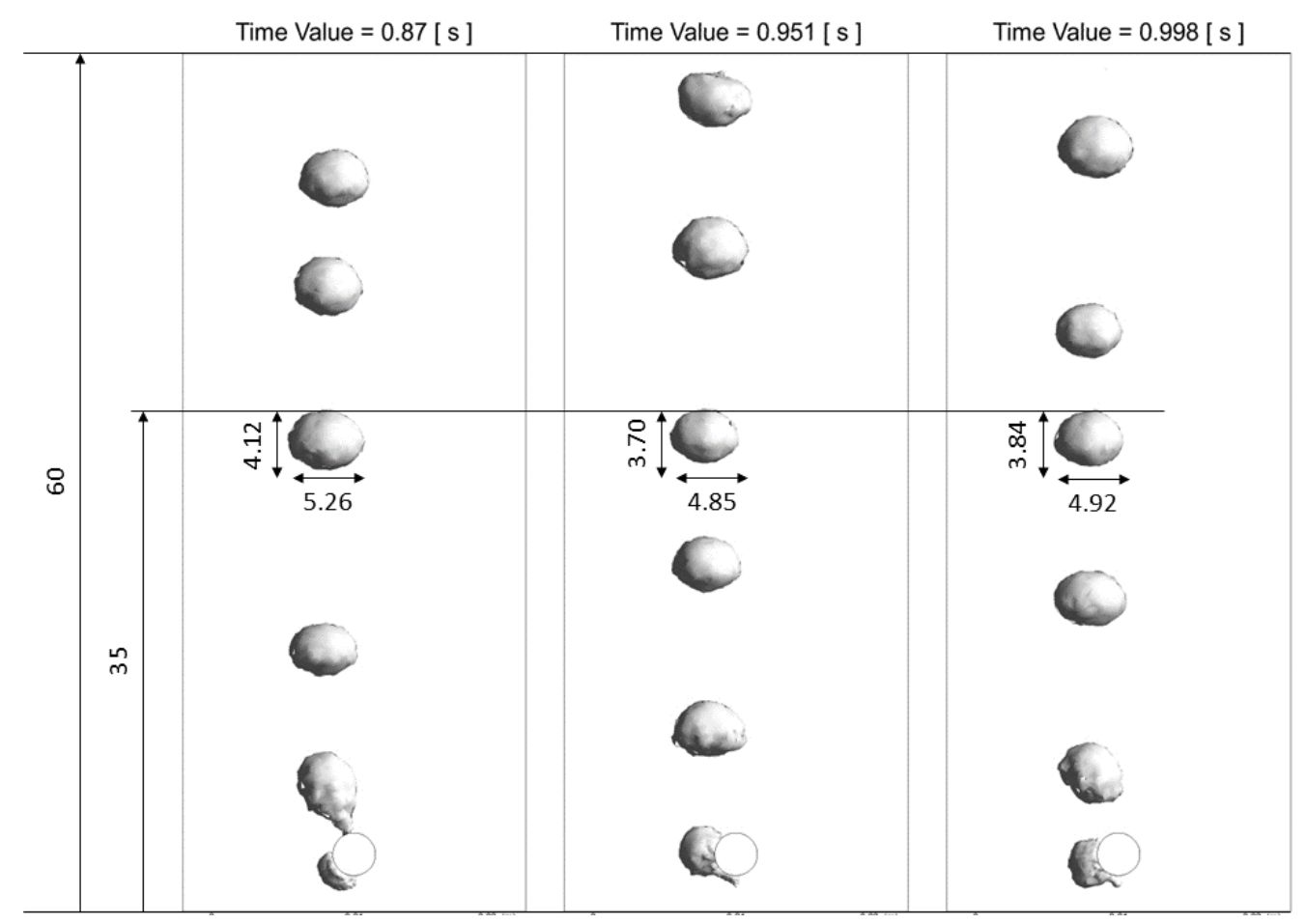

Fig. 3 Time snapshots of bubbles. Numerical analysis with the mass-source term. Shown as the iso-surface of volume fraction 0.5. All dimensions in $\mathrm{mm}$.

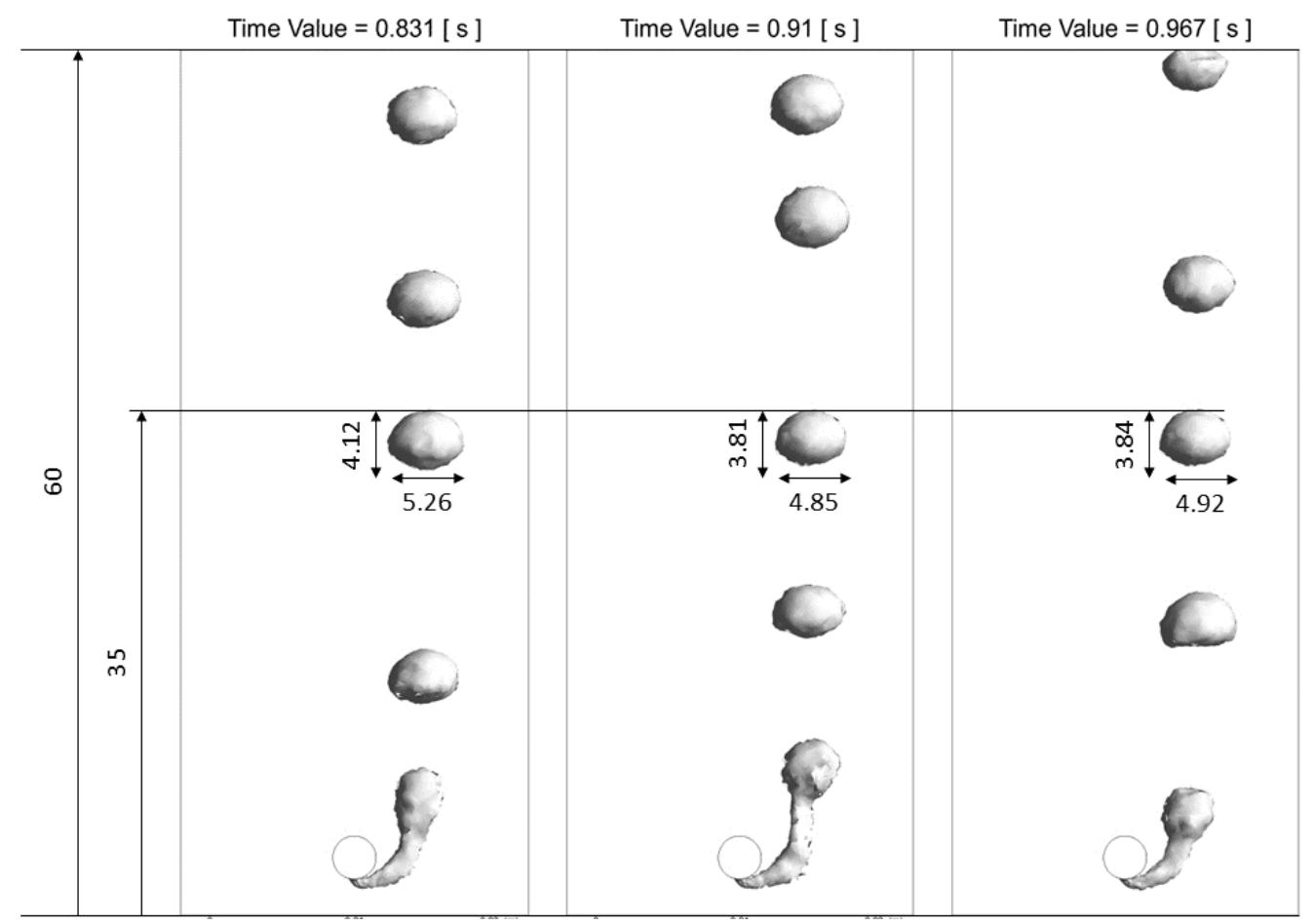

Fig. 4 Time snapshots of bubbles. Numerical analysis with the mass-flow-rate boundary condition. Shown as the iso-surface of volume fraction 0.5 . All dimensions in $\mathrm{mm}$. 
Tab. 2 summarizes the horizontal and vertical diameter together with the bubble aspect ratios for both simulated cases. The equivalent diameter according to Eq. (4) is $4.60 \mathrm{~mm}$ for the case with the mass-source term and $4.62 \mathrm{~mm}$ for the case with the mass-flow-rate boundary condition. The values are taken from the image analysis of the bubbly flow.

Tab. 2 Bubble diameters (in mm)

\begin{tabular}{cccccc} 
& Time, $\mathrm{s}$ & $d_{v}$ & $d_{h}$ & $d_{B}$ & Aspect ratio \\
\hline \multirow{3}{*}{ Mass-source term } & 0.870 & 4.12 & 5.26 & 4.85 & 0.78 \\
& 0.951 & 3.70 & 4.85 & 4.43 & 0.76 \\
& 0.998 & 3.84 & 4.92 & 4.53 & 0.78 \\
\hline \multirow{3}{*}{ Mass-flow-rate } & 0.831 & 4.12 & 5.26 & 4.85 & 0.78 \\
& 0.910 & 3.81 & 4.85 & 4.47 & 0.79 \\
& 0.967 & 3.84 & 4.92 & 4.53 & 0.78 \\
\hline
\end{tabular}

The bubble rise velocity was evaluated by means of contour plots showing instantaneous velocity fields at specific time steps (Fig. 5 and Fig. 6) and by tracking bubbles that flow through the domain. The tracking was based on processing time snapshots where bubbles were modelled as iso-surfaces set to volume fraction of 0.5 . In both cases, three bubbles were tracked starting at $0.55 \mathrm{~s}$ for the case with the mass-source term and $0.62 \mathrm{~s}$ for the case with the mass-flow-rate boundary condition. Resulting average bubble velocity was $0.175 \mathrm{~m} / \mathrm{s}$ for the setup with the mass-source term and $0.190 \mathrm{~m} / \mathrm{s}$ for the mass-flow-rate boundary condition.

Both flows are defined similarly in terms of the Eötvös and Morton numbers giving Eo $=2.9$ and $M o=2.6 \cdot 10^{-11}$, respectively. However, different average velocities yield $R e=804$ and $R e=876$ for the mass-source term and the massflow-rate boundary condition, respectively.

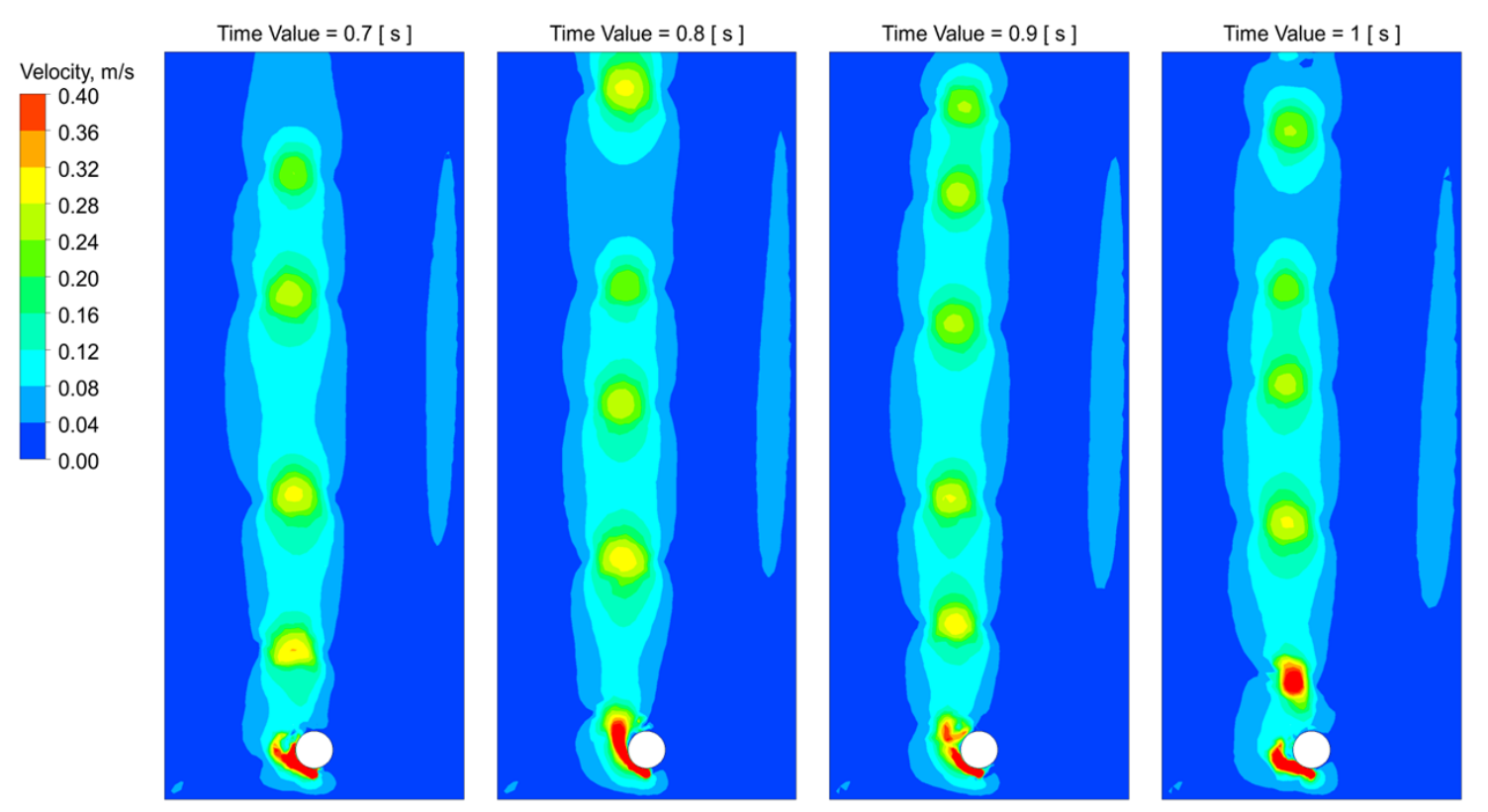

Fig. 5 Velocity contour plots. Numerical analysis with the mass-source term. Velocity range is limited to $0.4 \mathrm{~m} / \mathrm{s}$. 

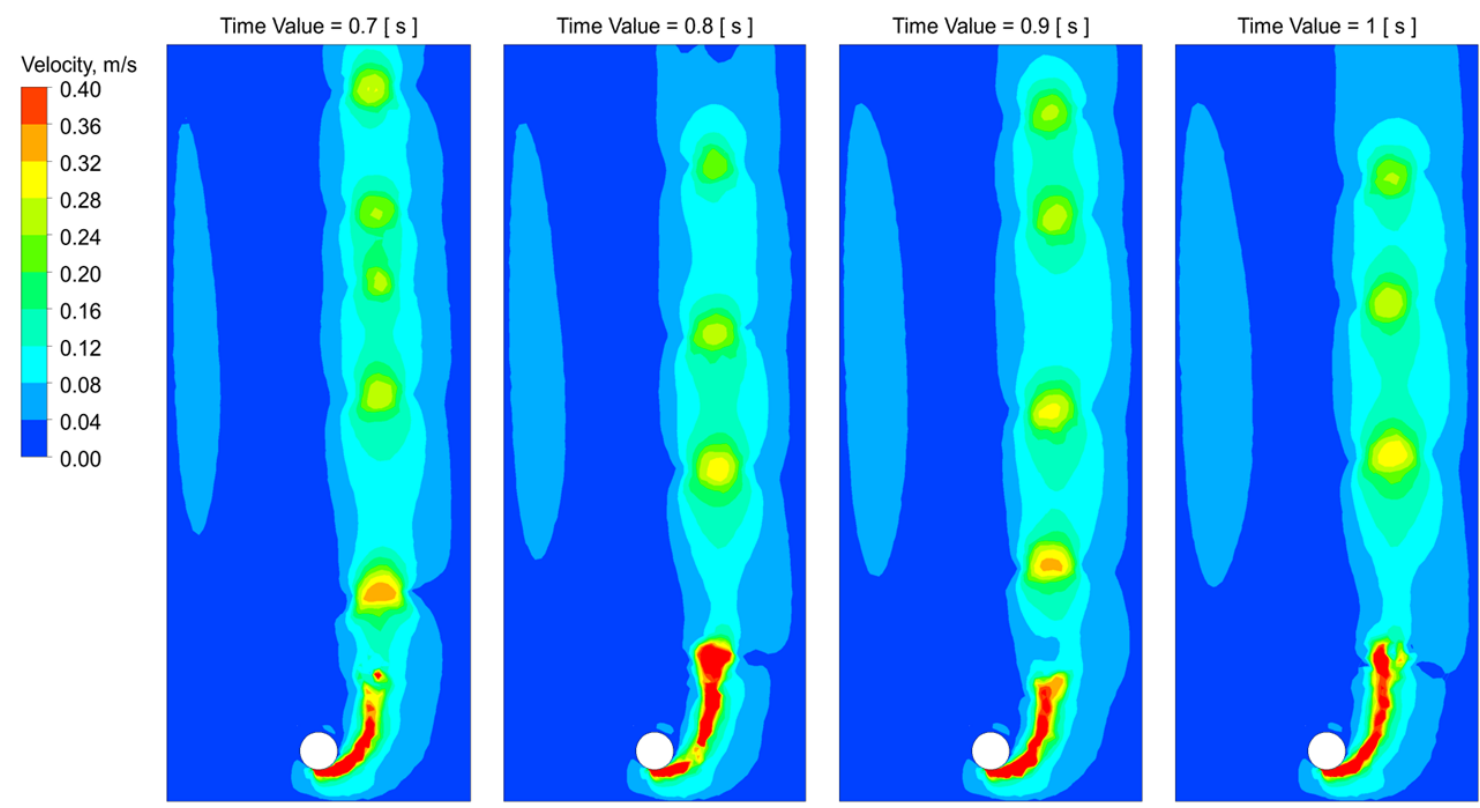

Fig. 6 Velocity contour plots. Numerical analysis with the mass-flow-rate boundary condition. Velocity range is limited to $0.4 \mathrm{~m} / \mathrm{s}$.

\section{Conclusions}

This paper has investigated the effect of air injection into the domain with a quiescent water. Two approaches were analysed where the injection of the gaseous phase was modelled. The first case used a mass-source term in a dedicated cell zone whereas the second case followed the real-world set-up with the mass-flow-rate boundary condition applied on a small part of the aerator tube. To keep this study comparative, both numerical analyses use one computational mesh for considered air injection modelling approaches.

The results of tracking the interphase area revealed the same bubble sizes, regardless of the air injection method. Therefore, flows in both numerical analyses follow the same definition of a bubbly flow in terms of the Eötvös and the Morton number. Also, based on the visual inspection of bubble shapes and comparison of aspect ratios, the shape of bubbles seems independent of the air injection method. A significant simplification in the case with the mass-source term was that the velocity gradient at the injection point was neglected. This may have resulted in a lower bubble rising velocity yielding also the difference in the Reynolds number.

Another observed difference in the considered modelling approaches was found to be the position of bubble separation (Fig. 3 and Fig. 4). In case of the mass-flow-rate boundary condition, separation of bubbles from the plume was found to be as high as $16 \mathrm{~mm}$ from the bottom surface. On the other hand, the bubble separation with the definition of the mass-source term takes place in a close proximity to the mass-source zone and the aerator tube.

Nevertheless, the study shows that the applicability of the mass-source term may be beneficial for subsequent numerical analyses of the complete photobioreactor. The definition of the mass-source term may allow to leave out the small inlet orifices so that the computational mesh will reach higher quality. 


\section{Acknowledgements}

This research was supported by the EU project Strategic Partnership for Environmental Technologies and Energy Production, funded as project No. CZ.02.1.01/0.0/0.0/16_026/0008413 by Czech Republic Operational Programme Research, Development and Education, Priority Axis 1: Strengthening capacity for high-quality research. Miroslav Rebej is Brno Ph.D. Talent Scholarship Holder - Funded by the Brno City Municipality.

\section{References}

[1] O. Pulz, 'Photobioreactors: production systems for phototrophic microorganisms', Appl. Microbiol. Biotechnol., vol. 57 , no. 3, pp. 287-293, Oct. 2001, doi: 10.1007/s002530100702.

[2] X. Gao, B. Kong, and R. D. Vigil, 'Simulation of algal photobioreactors: recent developments and challenges', Biotechnol. Lett., vol. 40, no. 9-10, pp. 1311-1327, Oct. 2018, doi: 10.1007/s10529-018-2595-3.

[3] M. J. Barbosa, Hadiyanto, and R. H. Wijffels, 'Overcoming shear stress of microalgae cultures in sparged photobioreactors', Biotechnol. Bioeng., vol. 85, no. 1, pp. 78-85, 2004, doi: https://doi.org/10.1002/bit.10862.

[4] L. Nedbal, M. Trtílek, J. Červený, O. Komárek, and H. B. Pakrasi, 'A photobioreactor system for precision cultivation of photoautotrophic microorganisms and for high-content analysis of suspension dynamics', Biotechnol. Bioeng., vol. 100, no. 5, pp. 902-910, 2008, doi: 10.1002/bit.21833.

[5] J. P. P. Bitog, I.-B. Lee, H.-M. Oh, S.-W. Hong, I.-H. Seo, and K.-S. Kwon, 'Optimised hydrodynamic parameters for the design of photobioreactors using computational fluid dynamics and experimental validation', Biosyst. Eng., vol. 122, pp. 42-61, Jun. 2014, doi: 10.1016/j.biosystemseng.2014.03.006.

[6] S. Almani, W. Blel, E. Gadoin, and C. Gentric, 'Investigation of single bubbles rising in Newtonian and non-Newtonian fluids inside a thin-gap bubble column intended for microalgae cultivation', Chem. Eng. Res. Des., vol. 167, pp. 218230, Mar. 2021, doi: 10.1016/j.cherd.2021.01.010.

[7] T. Ziegenhein and D. Lucas, 'Observations on bubble shapes in bubble columns under different flow conditions', Exp. Therm. Fluid Sci., vol. 85, pp. 248-256, Jul. 2017, doi: 10.1016/j.expthermflusci.2017.03.009.

[8] C. Thobie, E. Gadoin, W. Blel, J. Pruvost, and C. Gentric, 'Global characterization of hydrodynamics and gas-liquid mass transfer in a thin-gap bubble column intended for microalgae cultivation', Chem. Eng. Process. Process Intensif., vol. 122, pp. 76-89, Dec. 2017, doi: 10.1016/j.cep.2017.10.009.

[9] W. Dijkhuizen, M. van Sint Annaland, and J. A. M. Kuipers, 'Numerical and experimental investigation of the lift force on single bubbles', Chem. Eng. Sci., vol. 65, no. 3, pp. 1274-1287, Feb. 2010, doi: 10.1016/j.ces.2009.09.084.

[10]G. Bozzano and M. Dente, 'Shape and terminal velocity of single bubble motion: a novel approach', Comput. Chem. Eng., vol. 25, no. 4, pp. 571-576, May 2001, doi: 10.1016/S0098-1354(01)00636-6.

[11]ANSYS Inc., ANSYS Fluent Theory Guide, Release 2020 R2. Canonsburg, PA, USA: ANSYS Inc., 2020. 